\title{
Effect of temperature on field emission from a micrometer-long single-walled carbon nanotube
}

\author{
Chun-Sheng Wan, Zhen-Hua Li, and Kang-Nian Fan* \\ Shanghai Key Laboratory of Molecular Catalysis and Innovation Materials, Department of Chemistry, Centre for Chemical Physics, \\ Fudan University, Shanghai 200433, People's Republic of China
}

Xiao Zheng and Guan-Hua Chen*

Department of Chemistry, University of Hong Kong, Hong Kong, People's Republic of China

(Received 27 January 2006; revised manuscript received 17 March 2006; published 26 April 2006)

\begin{abstract}
Charge distribution along a micrometer-long carbon nanotube subject to an external applied electric field at different temperatures is determined via a quantum mechanical method. Corresponding emission current is evaluated via the Wentzel-Kramere-Brillouin approximation. For a capped micrometer-long $(5,5)$ nanotube, an external field of $10 \mathrm{~V} / \mu \mathrm{m}$ or above is required to produce the measurable current. Because of the overwhelming effects of the accumulation charges of the tip, the temperature alone has small effects on the field emission that can be observed in the experiments, and the strong self-heating effects are thus attributed mainly to the induced structural changes of carbon nanotubes.
\end{abstract}

DOI: 10.1103/PhysRevB.73.165422

\section{INTRODUCTION}

Research on field-emitting carbon nanotubes (CNTs) has attracted much attention ever since de Heer et al.'s first observation in $1995 .{ }^{1}$ Because of their high aspect ratio as well as mechanical and chemical stability, CNTs are regarded as potential materials for field emitters. It has been demonstrated that field emission from CNTs has low threshold voltage and high current density. For instance, the turn-on field of the CNT films can be as low as $5 \mathrm{~V} / \mu \mathrm{m}$, and CNT films can produce current density up to a few $\mathrm{A} / \mathrm{cm}^{2}$ under an external field below $10 \mathrm{~V} / \mu \mathrm{m} .{ }^{2,3}$ Thus the field emission of CNTs has been widely investigated in the experiments. ${ }^{4-7}$ It has been found that the emission current is saturated at higher applied voltages, ${ }^{8-10}$ and the defects on the tube body may affect the stability of the emission process.

Recently self-heating of CNTs during the field emission was observed, and the temperature can be heated up to $2000 \mathrm{~K}$ at the tips. The self-heating was attributed to the structure changes, ${ }^{11-13}$ and was observed to have significant influence on the field emission. ${ }^{14}$ Two questions thus arise. (1) How does the self-heating induce the structural changes and how do to the structure changes affect the field emission? Several theoretical works have been carried out to investigate the mechanism of self-heating and subsequent field emission modification. (2) How does the temperature alone affect the thermal distribution of electrons and thus results in the change of field emission current? Our work focuses on the second question. In our calculation, the structure of the tip is fixed during the calculations.

Two types of theoretical methods have been employed to simulate the field emission from CNTs. One is based on the dynamic field emission model, where the time-dependent Schrödinger equation is solved to simulate directly the emission process. ${ }^{15}$ This type of method is expected to be accurate but rather time consuming. The other is based on the static field emission model. ${ }^{16-21}$ The induced charge distribution under the external field is calculated upon the assumption that the field-emitting CNT is in quasi-equilibrium with the cathode substrate. The potential barrier for emission is
PACS number(s): 68.37.Vj, 61.46.Fg

subsequently determined and the emission current is estimated by the Wentzel-Kramere-Brillouin (WKB) approximation. We adopt the latter method in our present work.

Density functional theory calculations have been employed to investigate the field emission from CNTs. The basic model used is a short CNT saturated by hydrogen atoms or a fullerene-based cap at its end under an applied external field. Limited by computational efficiency and resources, only a few $\mathrm{nm}$ in length of CNT tip is considered explicitly. ${ }^{16-20}$ An underlying assumption is that the external field can be scaled to reproduce the correct local electrostatic field around the tube tip. However, it is doubtful whether the shortened tubes can mimic the realistic experiments. ${ }^{21}$ Therefore, it is desirable to include the entire tube in the simulation. This has been achieved by a hybrid quantummechanics/molecular-mechanics (QM/MM) approach developed by Zheng et al. ${ }^{22}$

\section{METHODOLOGY}

\section{A. Basic model}

To simulate field emission from CNTs, a 1- $\mu$ m-long $(5,5)$ capped single-walled carbon nanotube (SWNT) is taken as the model system in our calculation, which contains more than $10^{5}$ carbon atoms. The experimental setup is illustrated in Fig. 1. The hybrid QM/MM approach, which is widely employed in the research of the biological systems, is adopted to simulate the entire tube. ${ }^{23}$ The tip region containing a few thousand atoms is simulated via an explicit QM method while the rest is treated by a MM method. The interaction between the QM and MM parts is dominated by the electrostatic component. In our work, about 4000 atoms near the tip of the SWNT are assigned as the QM part and all other carbon atoms constitute the MM part. As Fig. 1 shows, the SWNT is connected to a metal cathode, and the joint system (the SWNT and the cathode) can be regarded as in quasi-equilibrium before the electrons emit or when the emission is not very strong. Therefore, the chemical potential or Fermi level should be a constant throughout the joint sys- 


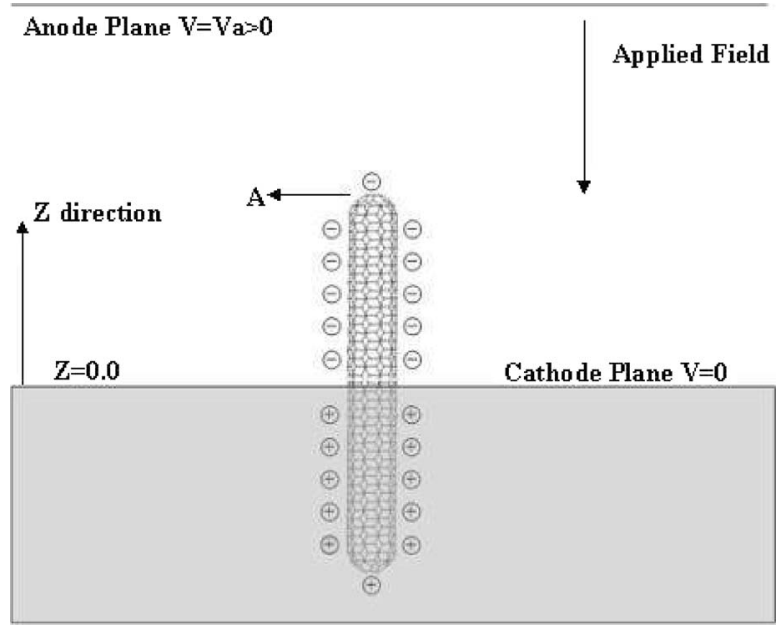

FIG. 1. The model of field emission from a SWNT. The gray part is the mirror of the nanotube, the potential of the cathode plane is set as zero. Negative induced charge accumulates on the surface of the nanotube. The zero of the coordinates of $z$ axis is set at the cathode plane and the zero of the $x$ axis is the central axis of the nanotube.

tem, and is equal to that of the bulk cathode. Due to the presence of the external field, excess electrons are injected from the cathode to the SWNT, leaving an equal amount of positive charges on the metal cathode surface. To satisfy the boundary condition, mirror images of the induced electrons along the entire SWNT are introduced as depicted in Fig. 1 and their electrostatic potential on the tube is taken into account. Note the point A indicates the very tip of the tube.

In our simulation, the divide-and-conquer method ${ }^{24}$ was employed to calculate the charge distribution in the QM region, and the modified neglect of diatomic overlap approximation $^{25}$ was adopted for the QM calculation. The induced charge distribution in the MM region was determined based on the Thomas-Fermi theory. The energies of the local orbitals are lowered upon the application of the external field. Some of them are lowered below the Fermi level, and are occupied subsequently by the induced electrons from the cathode. The number of these local orbitals is proportional to the potential energy shift $\delta V$. This is because the density of states nears the Fermi level is approximately constant, which has been validated by theoretical calculations $^{26}$ and experiments. ${ }^{27}$ Combined with the Poisson's equation $\nabla^{2} \delta V(z) \propto n(z)$, the induced charge density (or excess charge per unit length) along the tube can be expressed as

$$
\rho\left(z^{\prime}\right)=\rho(L) \frac{\sinh \left(z^{\prime} / \lambda\right)}{\sinh (L / \lambda)},
$$

where $\lambda$ represents the characteristic decay length and $\rho(L)$ is the linear induced charge density at $z=L$ where is the intersection of the simulation of the induced charge and the induced charge density from the calculation of the QM method. To simplify the calculation, the intersection limit $L$ is set to $100 \AA$ from the tip point A. The decay length and $\rho(L)$ are to be determined by extrapolating the calculated

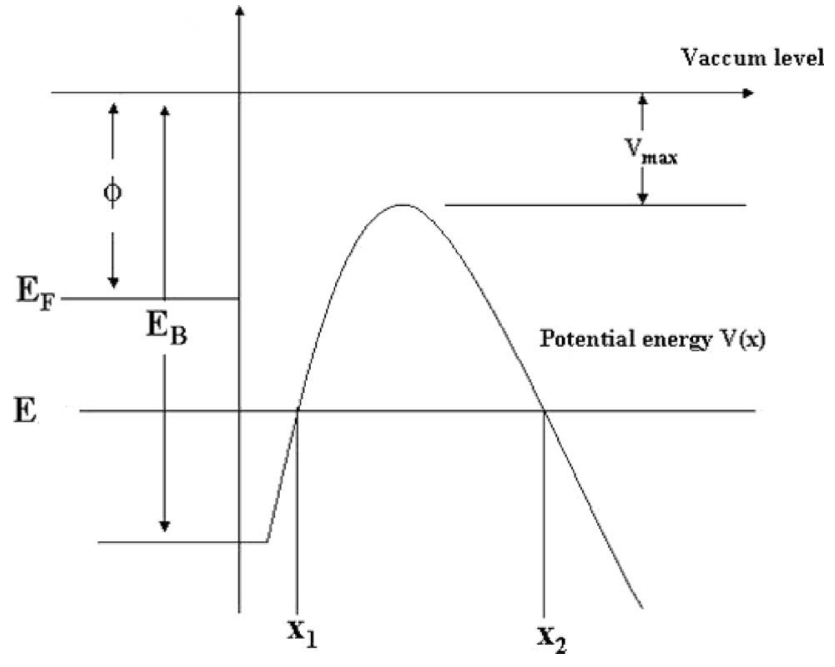

FIG. 2. The model of the classical field emission. The horizontal axis is the distance from the emitter surface. The region where $x$ is less than 0 means the inner part of the metal emitter and that where $x$ is larger than 0 means the outer part. The vertical axis is the potential barrier. The zero potential is set as the vacuum level. $\Phi$ is the work function of the emitters. $V(x)$ is the potential barrier near the emitter. $E_{F}$ is the Fermi level. $E_{B}$ is the binding energy of the emitter. $E$ is the total energy of the electrons. $V_{\max }$ is the maximum point of the barrier potential curve and $x_{1}, x_{2}$ are the coordinates of the point $V(x)$ equals $E$.

charge distribution in the QM region. For $T=298 \mathrm{~K}$ and the applied field equals $\mathrm{V} / \mu \mathrm{m}$, the decay length $\lambda$ and $\rho(L)$ are $0.44 \mu \mathrm{m}$ and $0.46 \mathrm{e} / \AA^{3}$. The QM region is in turn affected by the electrostatic potential field from the MM region. These procedures are repeated iteratively until $\rho(L)$ and $\lambda$ both converged. To ensure the computational accuracy, it was found that at least 2000 atoms should be contained in the QM region. In our calculation, a QM region having 4000 atoms is chosen. In the calculation of the electrostatic interaction between each subsystem and the environment, the effect of the QM part takes the charges of the atoms in last iteration and that of the MM part takes the simulation results.

\section{B. Temperature effect}

Due to the quasi-equilibrium approximation, the population of the conduction electrons on the metallic SWNT satisfies the Fermi-Dirac distribution. The traditional WKB approximation ${ }^{28}$ gives the transmission coefficient $D$ of the free electron gas as follows (Fig. 2):

$$
D(F, W)=\left[1+\exp \left(-2 i \hbar^{-1} \int_{x_{1}}^{x_{2}} p(x) d x\right)\right]^{-1} .
$$

And the transmission coefficient can be further simplified as

$$
D(F, W)=\left[1+\exp \left(-2 i \hbar^{-1} \int_{x_{1}}^{x_{2}} \sqrt{2 m(W-V(x)} d x\right)\right]^{-1},
$$

where $F$ is the applied external field, $W$ is the total energy of the free electron; $P$ is the momentum of the electron; $V(x)$ is 
(a)
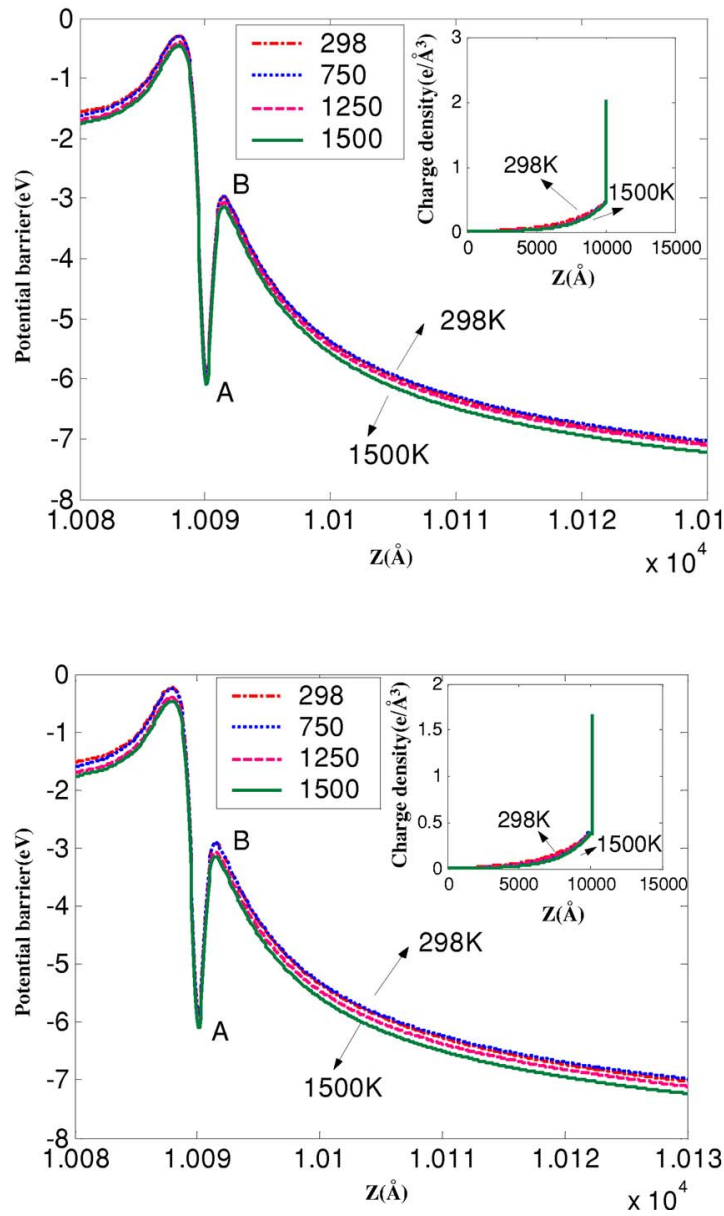
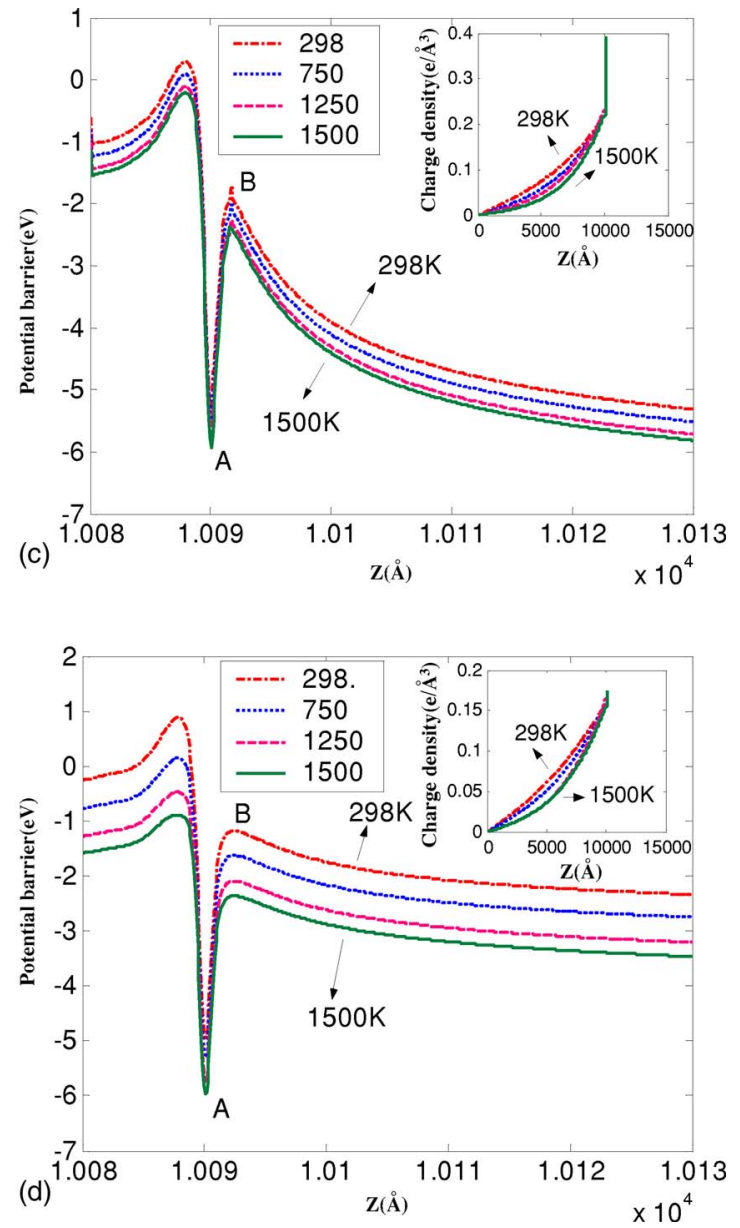

FIG. 3. (Color online) Induced charge density and electrostatic potential along the surface for the (5,5) $\mathrm{CNT}$. (a) $E_{\text {appl }}=10 \mathrm{~V} / \mu \mathrm{m}$; (b) $E_{\text {appl }}=7.5 \mathrm{~V} / \mu \mathrm{m}$; (c) $E_{\text {appl }}=5 \mathrm{~V} / \mu \mathrm{m}$; (d) $E_{\text {appl }}=2.5 \mathrm{~V} / \mu \mathrm{m}$. The horizontal axis is the coordinate from the cathode plane. The vertical axis is the potential barrier. Data are extracted along the central axis of the carbon nanotube. The potential curve is $298 \mathrm{~K}$ (dash-dot line), $750 \mathrm{~K}$ (dotted line), $1250 \mathrm{~K}$ (dashed line) and $1500 \mathrm{~K}$ (solid line). A is the valley point and B is the peak point of the potential barrier. The insets are the induced charge density along the axis. The horizontal axis is the coordinate from the cathode plane. The vertical axis is the induced charge density $\left(\mathrm{e} / \AA^{3}\right)$. All charge densities in (a) and (b) are in units of electron $/ \AA^{3}$.

the potential for an incoming electron; and $x_{1}, x_{2}$ are the coordinates of the point $V(x)$ equals $W$. To keep $D$ as a real number, the current density $J$ needs to be evaluated separately on intervals $W<V_{\max }$ and $W>V_{\text {max }}$. Here $V_{\text {max }}$ represents the potential barrier peak near the tip region. If the electronic energy $W$ is larger than $V_{\max }$, the value of the transmission coefficient should be exactly unity. The overall emission current density is thus expressed as the product of the incoming electron flux density and the transmission coefficients, i.e.

$$
\begin{aligned}
J= & \frac{4 \pi e m k T}{\hbar^{3}} \int_{-\infty}^{V_{\max }} \ln \left[1+\exp \left(-\frac{W-E_{F}}{k T}\right)\right] \\
& \times \exp \left[-\frac{2 m}{\hbar^{2}} \int_{x_{1}}^{x_{2}} \sqrt{V(x)-W} d x\right] d W \\
& \times+\frac{4 \pi e m k T}{\hbar^{3}} \int_{V_{\max }}^{\infty} \ln \left[1+\exp \left(-\frac{W-E_{F}}{k T}\right)\right] d W,
\end{aligned}
$$

where the Fermi level $E_{\mathrm{F}}$ is set to $-4.5 \mathrm{eV}$, which is the
Fermi level of bulk tungsten. The current is calculated the current density of the transverse different points at the tip with the WKB model and then these values are averaged to obtain the total emission current.

\section{RESULTS AND DISCUSSION}

To analyze the pure temperature effect on the field emission of CNTs, simulations have been carried out for six different temperatures ranging from 298 to $1500 \mathrm{~K}$ and four different applied fields $(2.5-10 \mathrm{~V} / \mu \mathrm{m})$. The charge and potential distributions are obtained for each combination of the applied field and temperature. Figure 3 depicts the potential barrier along the tube $\operatorname{axis}(X=0, Y=0)$ in the vicinity of the tip under the external fields of $2.5-10 \mathrm{~V} / \mu \mathrm{m}$. To make the results more clear, four of six temperatures are selected at each field: $298 \mathrm{~K}$ (dash-dot line), $750 \mathrm{~K}$ (dotted line), $1250 \mathrm{~K}$ (dashed line) and $1500 \mathrm{~K}$ (solid line). The induced charge distribution along the entire tube at different temperatures is plotted in the insets. Figures 3(a), 3(b), 3(c), and 3(d) 
show the calculated results for $E_{a p p l}=10.0,7.5,5.0$ and $2.5 \mathrm{~V} / \mu \mathrm{m}$, respectively. The point A represents the end of the tip where $z=10090.0 \AA$. This is also the point where the potential barrier is the minimum. The induced charge density along the entire tube is shown in the insets. The potential barrier is clearly lowered compared to the work function of the joint system, which implies the existence of strong field penetrations at the tip. From 298 to $1500 \mathrm{~K}$, the maximum variation of the potential barrier is less than $0.1 \mathrm{eV}$ in the tip region under the external field $10 \mathrm{~V} / \mu \mathrm{m}$, which means that the temperature has little effect on the strong applied field. At the external field $7.5 \mathrm{~V} / \mu \mathrm{m}$, the maximum variation of the potential barrier is less than $0.3 \mathrm{eV}$. As the field is lowered to 5.0 and $2.5 \mathrm{~V} / \mu \mathrm{m}$, the potential barrier has significant shift as temperature varies from 298 to $1500 \mathrm{~K}$. Maximum variations of 0.6 and $1.0 \mathrm{eV}$ for the potential barrier are observed. The insets of Figs. 3(c) and 3(d) indicate that the induced charge distribution varies along the tube as the temperature changes for the low external field. While the insets of Figs. 3(a) and 3(b) show that the induced charge distribution varies little as the temperature changes for the strong applied fields. Figure 4 shows clearly the lowering of the potential barrier, which is the potential at B (the highest potential barrier in the tip region) versus the Fermi level, with respect to the increasing temperature for various applied external fields. The rate of the potential barrier change with respect to the temperature is larger at lower fields. To investigate the effects for the potential barrier of the whole tube, Figs. 5(a)-5(d) show the potential energy contour plots for two temperatures: 298 and $1500 \mathrm{~K}$ for four different fields $E_{a p p l}$ $=10 \mathrm{~V} / \mu \mathrm{m}$ to $E_{\text {appl }}=2.5 \mathrm{~V} / \mu \mathrm{m}$, respectively. The change in potential field distribution with respect to the temperature can be identified by comparing different panels. More changes are found at the low fields than high fields. The largest change of the potential barrier with temperature is no larger than $0.1 \mathrm{eV}$. That indicates most of the changes gathered at the tip region.

With the knowledge of the local electric field distribution, the emitting current intensity and the local field can be calculated via Eq. (4) and the results are shown in Table I. In the table, $E_{a p p l}$ stands for the applied external field and $E_{l o c}$ is the average local field intensity with respect to the increasing temperature, which is calculated by the derivate of potential barrier along the tube axis near the tip region. The average size is $5 \AA$ from the tip point. As Table I shows, the emission

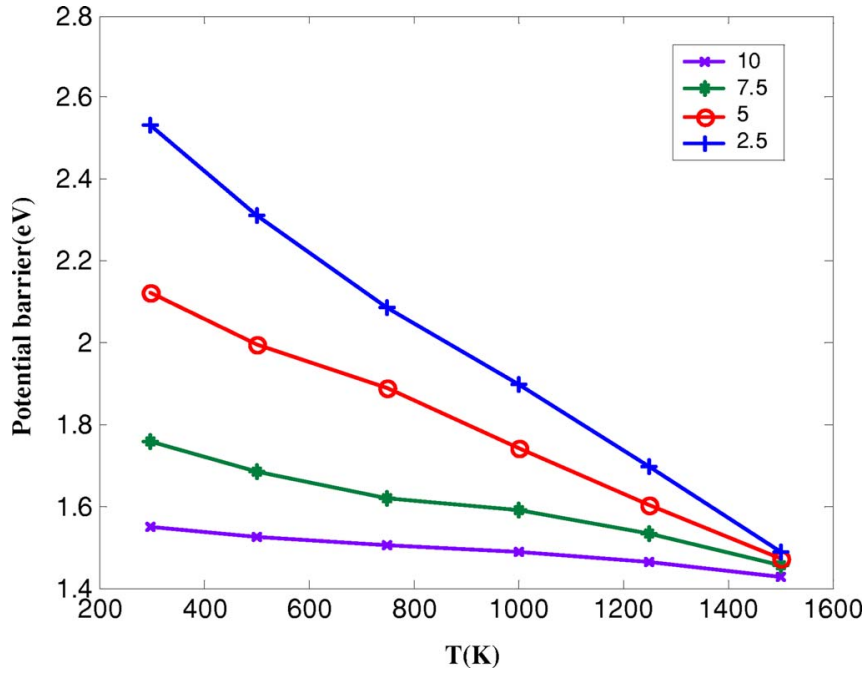

FIG. 4. (Color online) Potential barrier above Fermi level under different applied fields $(\mathrm{V} / \mu \mathrm{m})$. The horizontal axis is the different temperature. The vertical axis is the relative potential barrier $(\mathrm{eV})$ of the surface. The value equals the minus of the potential barrier of the peak point (B) and Fermi level $E_{\mathrm{F}}(-4.5 \mathrm{eV})$.

current increases less than twice from 298 to $1500 \mathrm{~K}$ for $E_{a p p l}=10 \mathrm{~V} / \mu \mathrm{m}$, which indicates that the current intensity depends slightly on the temperature when the applied field is as high as $10 \mathrm{~V} / \mu \mathrm{m}$. This is because the strong applied field induces an excessive amount electrons in the tip region, which leads to the strong local field that overwhelms the temperature effects. The magnitude of the current intensity shrinks drastically as $E_{a p p l}$ decreases, while at the same time, the influence of temperature on the current intensity becomes more and more significant. For instance, the current intensity at $1500 \mathrm{~K}$ is about 4 , more than 20 , more than ten thousand times of its value at $298 \mathrm{~K}$ for $E_{a p p l}=7.5,5.0$, and $2.5 \mathrm{~V} / \mu \mathrm{m}$, respectively. The changes of the potential barrier have obvious influence on the emission current. Note that from Table I the local field intensity at the tip changes little as the temperature varies under a fixed applied field. This phenomenon is due to the fact that the induced charge distribution at the tip region varies little as the temperature changes.

The results obtained above can be interpreted based on the quasi-equilibrium approximation. Upon this approximation, the Fermi level of the electrons on the SWNT equals the

TABLE I. Local field of the central axis $\left(E_{l o c}\right)$ and current intensity $(I)$ for the $(5,5)$ CNT at four fields.

\begin{tabular}{|c|c|c|c|c|c|c|c|c|}
\hline \multirow[t]{2}{*}{$T / K$} & \multicolumn{2}{|c|}{10} & \multicolumn{2}{|c|}{7.5} & \multicolumn{2}{|c|}{5} & \multicolumn{2}{|c|}{2.5} \\
\hline & $\begin{array}{c}E_{l o c} \\
\left(10^{6} \mathrm{~V} / \mathrm{cm}\right)\end{array}$ & $\begin{array}{c}I \\
\left(10^{-9} \mathrm{~A}\right)\end{array}$ & $\begin{array}{c}E_{l o c} \\
\left(10^{6} / \mathrm{cm}\right)\end{array}$ & $\begin{array}{c}I \\
\left(10^{-18} \mathrm{~A}\right)\end{array}$ & $\begin{array}{c}E_{l o c} \\
\left(10^{6} \mathrm{~V} / \mathrm{cm}\right)\end{array}$ & $\begin{array}{c}I \\
\left(10^{-41} \mathrm{~A}\right)\end{array}$ & $\begin{array}{c}E_{l o c} \\
\left(10^{6} \mathrm{~V} / \mathrm{cm}\right)\end{array}$ & $\begin{array}{c}I \\
\left(10^{-87} \mathrm{~A}\right)\end{array}$ \\
\hline 298 & 36.61 & 4.92 & 21.21 & 2.15 & 9.05 & 6.48 & 4.31 & 9.67 \\
\hline 500 & 36.62 & 5.22 & 21.25 & 2.82 & 9.09 & 13.71 & 4.37 & 36.53 \\
\hline 750 & 36.77 & 5.61 & 21.37 & 3.71 & 9.00 & 24.84 & 4.30 & 171.55 \\
\hline 1000 & 37.46 & 6.18 & 20.95 & 5.13 & 9.00 & 47.70 & 4.36 & 1038.76 \\
\hline 1250 & 37.26 & 6.81 & 21.53 & 6.49 & 9.08 & 87.58 & 4.30 & 11512.72 \\
\hline 1500 & 37.05 & 7.68 & 21.43 & 8.38 & 9.21 & 170.27 & 4.33 & 15672.18 \\
\hline
\end{tabular}


(a)
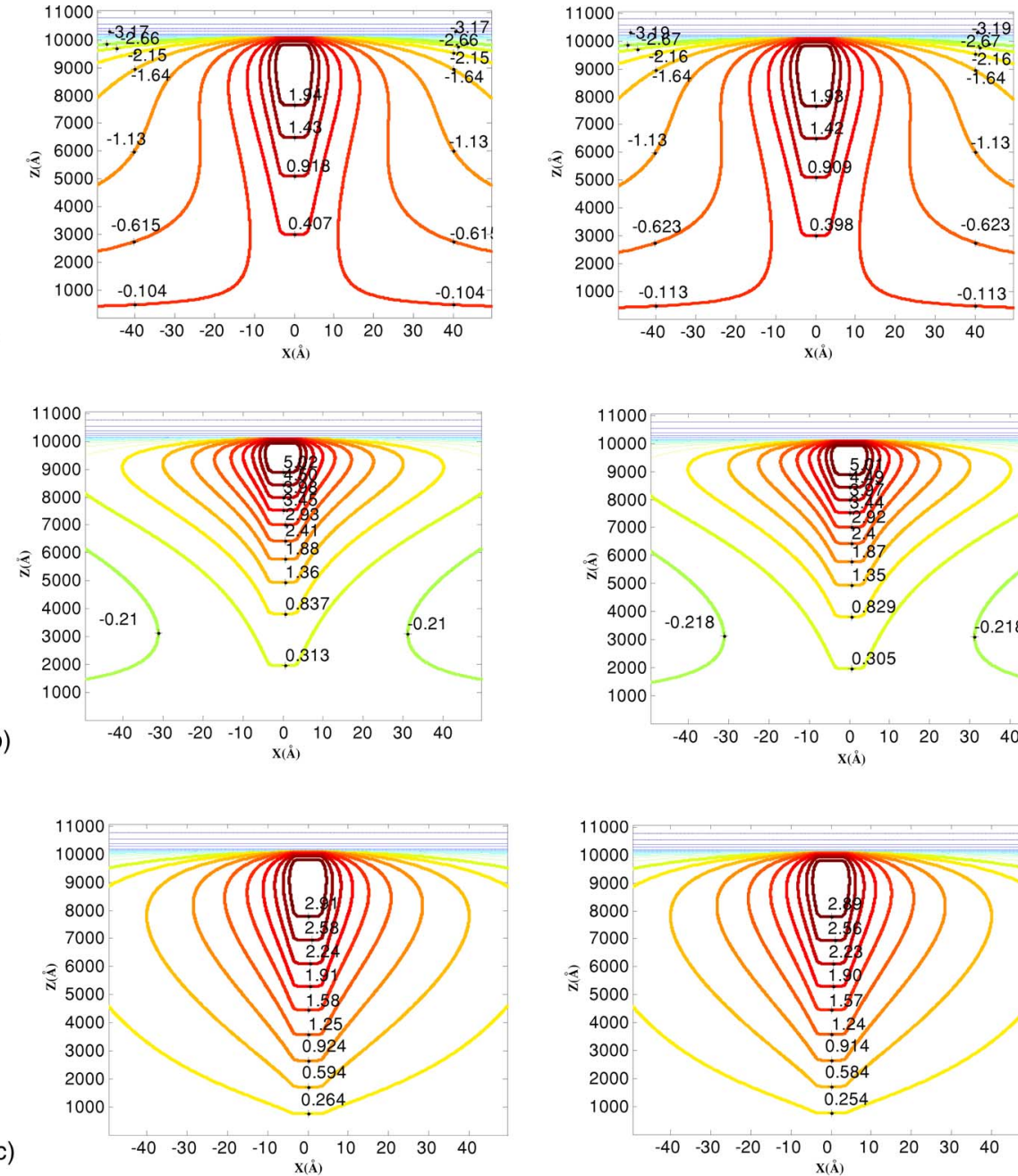

(c)

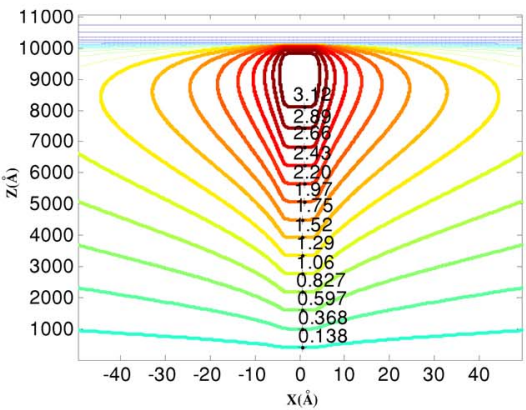

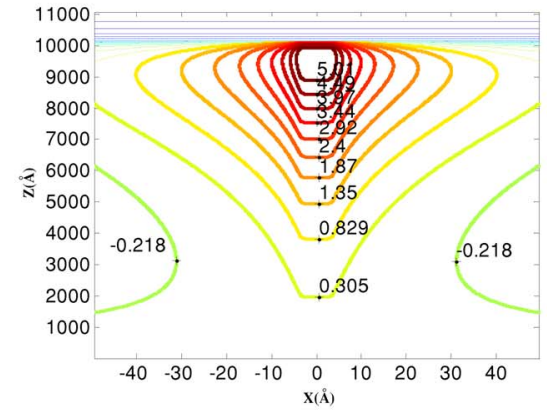
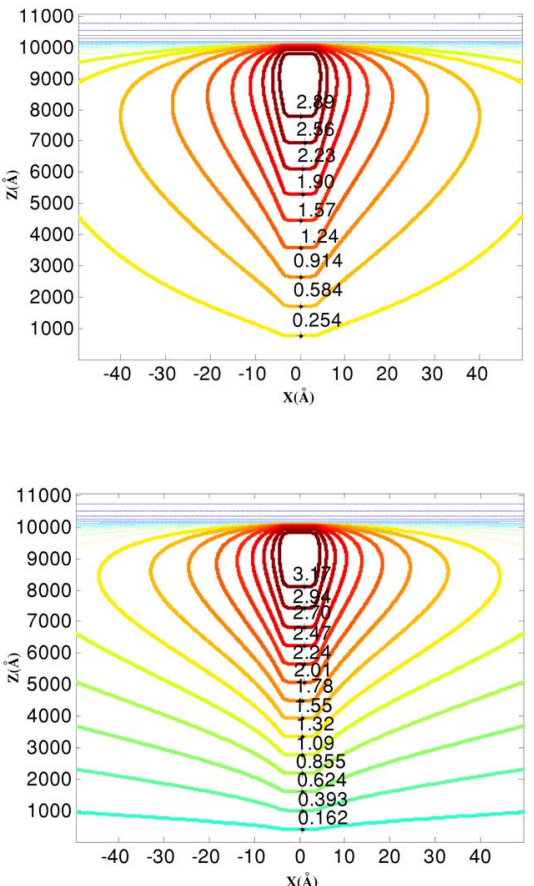

FIG. 5. (Color online) Potential energy contour plots for the $(5,5)$ CNT. (a) $E_{\text {appl }}$ $=10 \mathrm{~V} / \mu \mathrm{m}, \quad T=298$ and $1500 \mathrm{~K}$; (b) $E_{\text {appl }}=7.5 \mathrm{~V} / \mu \mathrm{m}$, $T=298$ and $1500 \mathrm{~K}$. (c) $E_{\text {appl }}$ $=5 \mathrm{~V} / \mu \mathrm{m}, \quad T=298$ and $1500 \mathrm{~K}$; (d) $E_{\text {appl }}=2.5 \mathrm{~V} / \mu \mathrm{m}$, $T=298$ and $1500 \mathrm{~K}$. The zero of the horizontal axis is the central axis. The vertical axis is the coordinate from the cathode plane. chemical potential of the cathode, which is set to $-4.5 \mathrm{eV}$ in the calculations. The electron distribution obeys the FermiDirac distribution and is affected by the change of temperature. At higher temperature, the electrons have more probability to be thermally excited to the vacant local orbitals at the tip region. This leads to the induced electrons to gather at the tip region. Due to the accumulation of induced electrons at the tip, the enhanced positive surrounding at the tip results in the lowered potential barrier. The influence of these changes is different for various applied fields. At a highapplied field (e.g., $10 \mathrm{~V} / \mu \mathrm{m}$ ) the electron density and the local field at the tip region are very high. As a result, the thermal effects become secondary. The relative change of the current intensity is thus small. At a low applied field, the induced electron density and the local field are relatively low. The thermal effects thus become more important. The tunnel probability is larger at high temperature. This is advantageous to the field emission process. In addition, as we noticed before, the local field intensity changes little at various temperatures. This is due to the fact that the local field mainly depends on the charge distribution at the tip region and the electron distribution at this region is almost unaltered at different temperatures. At a low field, the induced charge distribution away from the tip region varies as the tempera- 
ture changes. However, this variation affects only the overall value of the potential barrier but not the shape of the potential curve since these charges are quite far away. As a consequence, the local field intensity remains similar. Compared with the calculated emission current and the emission current measured in the experiments, ${ }^{3-10}$ only the current intensity from our $(5,5) \mathrm{SWNT}$ at $10 \mathrm{~V} / \mu \mathrm{m}$ can be detected under the realistic experimental conditions. Although our calculations show that the emitting current intensity is affected significantly by the temperature below $7.5 \mathrm{~V} / \mu \mathrm{m}$, the current intensity at these applied is negligible. At usual applied fields (more than $10 \mathrm{~V} / \mu \mathrm{m}$ ), the value of the emission current can be 100 or 1000 times the results at $10 \mathrm{~V} / \mu \mathrm{m}$. Under these conditions, more induced charges are accumulated at the tip, which leads to the dominating electrostatic effect over that of the pure temperature. Therefore, we conclude that for all practical purposes the pure temperature effect on CNT field emission intensity is small. The strong self-heating effects on CNT field emission should thus be attributed mainly to the induced structural changes.

\section{CONCLUSION}

Based on our calculations, it is found that the temperature alone has little effect on the field emission from CNTs. This is due to the overwhelming electrostatic effect from the induced charges at the tip region as compared to the pure temperature effect on the field emission. Although at very weak applied fields the pure temperature effect may become significant, the corresponding emitting current intensity is found to be too small to have any practical usages. The stronger self-heating influence on CNT emission should be mainly attributed to the induced structure modification. Work along this direction is in progress.

\section{ACKNOWLEDGMENTS}

This work was financially supported by the National Natural Science Foundation of China (200433020) and the National Science Foundation of Shanghai Science and Technology Committee (02DJ14023) and the Hong Kong Research Grant Council (HKU 7012/04P).
*Corresponding authors. Electronic address: knfan@fudan.edu.cn; ghc@everest.hku.hk

${ }^{1}$ W. A. de Heer, W. S. Bacsa, A. Chatelain, T. Gerfin, R. Humphrey-Baker, L. Forro, and D. Ugarte, Science 268, 845 (1995).

${ }^{2}$ J. M. Bonard, J. P. Salvetat, T. Stockli, and W. A. de Heer, Appl. Phys. Lett. 73, 918 (1998).

${ }^{3}$ X. P. Xu and G. R. Brandes, Appl. Phys. Lett. 74, 2549 (1999).

${ }^{4}$ P. Chen, X. Wu, X. Sun, J. Lin, W. Ji, and K. L. Tan, Phys. Rev. Lett. 82, 2548 (1999).

${ }^{5}$ R. Gao, Z. Pan, and Z. L. Wang, Appl. Phys. Lett. 78, 1757 (2001).

${ }^{6}$ J.-M. Bonard, K. A. Dean, B. F. Coll, and C. Klinke, Phys. Rev. Lett. 89, 197602 (2002).

${ }^{7}$ Z. L. Wang, R. P. Gao, W. A. de Heer, and P. Poncharal, Appl. Phys. Lett. 80, 856 (2002).

${ }^{8}$ R. B. Sharma, V. N. Tondare, D. S. Joag, A. Govindaraj, C. N. R. Rao, Chem. Phys. Lett. 344, 283 (2001).

${ }^{9}$ P. G. Collins and A. Zettl, Phys. Rev. B 55, 9391 (1997).

${ }^{10}$ K. A. Deana and B. R. Chalamala, Appl. Phys. Lett. 76, 375 (2000).

${ }^{11}$ P. Vincent, S. T. Purcell, C. Journet, and Vu Thien Binh, Phys. Rev. B 66, 75406 (2002).

${ }^{12}$ S. T. Purcell, P. Vincent, C. Journet, and Vu Thien Binh, Phys. Rev. Lett. 88, 105502 (2002).

${ }^{13}$ M. Sveningsson, K. Hansen, K. Svensson, E. Olsson, and B. Campbell1, Phys. Rev. B 72, 085429 (2005).
${ }^{14}$ Z. Xu, X. D. Bai, E. G. Wang, and Z. L. Wang, Appl. Phys. Lett. 87, 163106 (2005).

${ }^{15}$ S. Han, M. H. Lee, and J. Ihm, Phys. Rev. B 65, 085405 (2002).

${ }^{16}$ A. Mayer, N. M. Miskovsky, P. H. Cutler, and Ph. Lambin, Phys. Rev. B 68, 235401 (2003).

${ }^{17}$ Ch. Adessi and M. Devel, Phys. Rev. B 62, R13314 (2002).

${ }^{18}$ G. Zhou, W. Duan, and B. Gu, Phys. Rev. Lett. 87, 095504 (2001).

${ }^{19}$ S. Han and J. Ihm, Phys. Rev. B 61, 9986 (1999).

${ }^{20}$ Y. Gohda, Y. Nakamura, K. Watanabe, and S. Watanabe, Phys. Rev. Lett. 85, 1750 (1999).

${ }^{21}$ J. Luo, L.-M. Peng, Z. Q. Xue, and J. L. Wu, Phys. Rev. B 66, 155407 (2002).

${ }^{22}$ X. Zheng, G. H. Chen, Z. Li, S. Deng, and N. Xu, Phys. Rev. Lett. 92, 106803 (2004).

${ }^{23}$ A. Warshel and M. Levitt, J. Mol. Biol. 103, 227 (1976).

${ }^{24}$ W. Yang, Phys. Rev. Lett. 66, 1438 (1991).

${ }^{25}$ M. J. S. Dewar and W. Thiel, J. Am. Chem. Soc. 99, 4899 (1977).

${ }^{26}$ A. M. Rao, E. Richter, S. Bandow, B. Chase, P. C. Eklund, K. A. Williams, S. Fang, K. R. Subbaswamy, M. Menon, A. Thess, R. E. Smalley, G. Dresslhaus, and M. S. Dresselhaus, Science 275, 187 (1997); X. Blase, L. X. Benedict, E. L. Shirley, and S. G. Louie, Phys. Rev. Lett. 72, 1878 (1994); R. A. Jishi, J. Bragin, and L. Lou, Phys. Rev. B 59, 9862 (1999).

${ }^{27}$ J. W. G. Wildöer, L. C. Venema, A. G. Rinzler, R. E. Smalley, and C. Dekker, Nature (London) 391, 59 (1998).

${ }^{28}$ E. L. Murphy and R. H. Good, Phys. Rev. 102, 1464 (1956). 